\title{
More severe sustained attention deficits in nonpsychotic siblings of multiplex schizophrenia families than in those of simplex ones
}

\author{
Hui-Chun Tsuang ${ }^{\mathrm{a}}$, Sheng-Hsiang Lin ${ }^{\mathrm{a}}$, Shi K. Liu ${ }^{\mathrm{b}}$, Ming-Hsien Hsieh ${ }^{\mathrm{b}}$, \\ Tzung J. Hwang ${ }^{b}$, Chih-Min Liu ${ }^{b}$, Hai-Gwo Hwu ${ }^{\text {a,b }}$, Wei J. Chen ${ }^{\text {a,b,* }}$ \\ a Institute of Epidemiology, College of Public Health, National Taiwan University, 1 Jen-Ai Rd., Sec. 1, Taipei 100, Taiwan, ROC \\ ${ }^{\mathrm{b}}$ Department of Psychiatry, College of Medicine and National Taiwan University Hospital, National Taiwan University, Taipei, Taiwan, ROC
}

Received 6 September 2005; received in revised form 4 March 2006; accepted 24 March 2006

Available online 5 June 2006

\begin{abstract}
Sustained attention deficits measured by the Continuous Performance Test (CPT) have been proposed as an endophenotype of schizophrenia. However, little is known about whether sustained attention deficits in first-degree relatives of schizophrenic patients are associated with familial loading for schizophrenia. We examined 107 parents and 84 siblings of simplex schizophrenia families as well as 72 parents and 56 siblings of multiplex schizophrenia families, all nonpsychotic, using the Diagnostic Interview for Genetic Studies and two sessions of the CPT (undegraded and degraded). The effect of perceptual load was assessed using the residual of the regression of the degraded score on the undegraded one. Statistical models that can adjust for familial correlations were used to compare the CPT performance of relatives between the two types of families. Siblings from multiplex families exhibited worse performance on the degraded CPT and less proficiency in processing the perceptual load than those from simplex families. No such difference was observed for the parents on either CPT version. We concluded that sustained attention along with perceptual load processing is more impaired in the siblings of schizophrenic patients with high familial loading and that this finding might be useful for future genetic dissection of schizophrenia.
\end{abstract}

(C) 2006 Elsevier B.V. All rights reserved.

Keywords: Schizophrenia; Sustained attention deficits; Endophenotypes; Genetic loading; Simplex families; Multiplex families

\section{Introduction}

Heterogeneity in schizophrenia has been a central issue in the elucidation of its etiology (Tsuang et al.,

\footnotetext{
* Corresponding author. Institute of Epidemiology, College of Public Health, National Taiwan University, 1 Jen-Ai Rd., Sec. 1, Taipei 100, Taiwan, ROC. Tel.: +8862 23516480; fax: +886223560840.

E-mail addresses: hctsuang@ms14.hinet.net (H.-C. Tsuang), d90842002@ntu.edu.tw (S.-H. Lin), Formosa@ha.mc.ntu.edu.tw (S.K. Liu),msthsieh@ha.mc.ntu.edu.tw (M.-H. Hsieh), johnson@ha.mc.ntu.edu.tw (T.J. Hwang), liupan@ha.mc.ntu.edu.tw (C.-M. Liu), haigohwu@ha.mc.ntu.edu.tw (H.-G. Hwu), weijen@ha.mc.ntu.edu.tw (W.J. Chen).
}

1990). One way to tackle this is to identify a subgroup of patients with higher genetic loading on the basis of family history (Farmer et al., 1990; Lewis et al., 1987). The rationale is that patients with negative family history (sporadic cases) would have less genetic loading for schizophrenia than those with positive family history (familial cases). However, given the low penetrance of a disease gene, the distinction between sporadic and familial subtypes of disease is not straightforward (Kendler, 1987, 1988). Another concern is how to define the magnitude of genetic contribution in schizophrenic patients. One study comparing schizophrenic patients who did not have other affected siblings 
with those who did found no differences in their psychotic symptoms or other comorbidity except a lower prevalence of alcohol abuse in the latter group (DeLisi et al., 2001). If alcohol abuse was unrelated to the genetic vulnerability to schizophrenia, this finding implied that substances might have a substantial role in the development of schizophrenia in simplex families rather than genes and the patients from simplex families were indistinguishable clinically from those with considerable genetic contribution. It thus lends support to the potential utility of family history in terms of indicating genetic loading in schizophrenia.

On the other hand, the endophenotype approach has been increasingly adopted by researchers in the search for the genetic predisposition to schizophrenia (Almasy and Blangero, 2001; Gottesman and Gould, 2003). If patients are found to have the endophenotype, their nondiseased family members would have a higher rate of endophenotype expression than the general population. Among several candidates, sustained attention deficits as measured on the Continuous Performance Test (CPT) (Rosvold et al., 1956) have been proposed as an endophenotype of schizophrenia. CPT deficits have been shown to be present not only in schizophrenic patients, but also in subjects with schizotypal personality disorder as well as nonpsychotic relatives of schizophrenic patients (Chen and Faraone, 2000; Cornblatt and Keilp, 1994). In addition, using 2.5 standard deviations or more below the population mean as threshold, the risk ratios of CPT performance deficits among parents or siblings to that in the general population were higher than the risk ratio of schizophrenia alone (Chen et al., 1998b, 2004).

A combination of both approaches is to use some probable endophenotypes, which can be measured objectively even in non-diseased family members, to compare the families of sporadic with those of familial schizophrenia. To date, only two studies specifically compared the CPT performance of schizophrenic patients with positive family history versus those without, but their relatives were not examined (Roy et al., 1994; Walker and Shaye, 1982).

The objective of this study was to test a hypothesis that sustained attention deficit in nonpsychotic firstdegree relatives of schizophrenic patients would be worse in multiplex than in simplex families. Simplex families in this study are similar to so-called "sporadic" ones except that the affection status is limited to first-degree relatives. The definition of a multiplex case is more stringent than a familial one in the sense that the familiality is restricted to a co-affected sibling. The study also tested the following more specific hypotheses: (1) The difference between simplex and multiplex families in sustained attention deficit might be greater for siblings than for parents, since a sib-pair share one more component in genetic covariance than a parent-offspring pair (Falconer and Mackay, 1996). (2) A more difficult version of the CPT might reveal a greater difference between simplex and multiplex families in sustained attention deficit, since a higher recurrence risk ratio was observed using the degraded than the undegraded version of the CPT in nonpsychotic relatives of schizophrenic patients (Chen et al., 2004). (3) A component measured by the CPT other than sustained attention per se, i.e., the perceptual load component that can be isolated by regressing a relative's degraded score on his undegraded one, might distinguish simplex from multiplex families since deficiency in information processing is part of the proposition that neurocognitive deficits are involved in schizophrenia (Braff, 1993; Nuechterlein and Dawson, 1984).

\section{Methods}

\subsection{Probands}

Probands of both simplex and multiplex families were recruited from two studies on schizophrenia. The first study, the Multidimensional Psychopathology Group Research Project, aimed to recruit schizophrenic patients and their first-degree relatives. From August 1993 to June 1998, patients consecutively admitted to the acute inpatient wards of the National Taiwan University Hospital, the Taipei City Psychiatric Center and the Provincial Tao-Yuan Psychiatric Center were included if they met the DSM-III criteria for schizophrenic disorders (Chang et al., 2002; Chen et al., 1998b). During the study period, the diagnostic criteria were shifted to DSM-IV and earlier subjects were rediagnosed with the updated criteria. The second study, the Multidimensional Psychopathological Study on Schizophrenia, aimed to collect sib-pairs who were coaffected with schizophrenia and had at least two alive first-degree relatives. The affected sib-pair probands, who met the DSM-IV criteria for schizophrenia or schizoaffective disorders, depressed type, were identified from either the inpatient wards or outpatient clinics of the National Taiwan University Hospital and the Provincial Tao-Yuan Psychiatric Center from July 1998 to December 2001. Written informed consent was obtained from all subjects after complete description of the study. Both studies were approved by the institutional review boards of the participating hospitals.

On the basis of affection status among first-degree relatives, 11 out of the 91 families recruited from the 
first study (Chen et al., 2004) did not fulfill the definition of simplex families: six had an affected sibling (one had already been included in the second study and the remaining five families were reassigned to the multiplex group for subsequent analyses); another five families had one parent affected with schizophrenia or schizophreniform disorders and were excluded from this study. Because the schizophrenic probands in each multiplex family were not examined concurrently during their hospital admissions for the CPT, several factors have to be incorporated before subjecting their CPT performances to comparison with those of simplex families. Thus, the sustained attention deficits of these probands will be reported in a separate paper and the current study will be focused on relatives only.

\subsection{Measurements}

\subsubsection{Interview instruments and diagnostic procedures}

Probands and their first-degree relatives were interviewed with the Diagnostic Interview for Genetic Studies (DIGS) (NIMH Genetics Initiative, 1992a), which was designed specifically for family-genetic studies of schizophrenia and bipolar disorder with good inter-rater reliabilities (Nurnberger et al., 1994). Kappas for the Chinese version of the DIGS for the diagnoses of schizophrenia, bipolar disorder and major depression ranged from 0.86 to 0.93 (Chen et al., 1998b). Interviews with the Chinese version of the DIGS were carried out by research assistants who had received standardized psychiatric interview training. In addition to the DIGS, interviewers used the Chinese version of the Family Interview for Genetic Studies (FIGS) (NIMH Genetics Initiative, 1992b) to collect relevant information on relatives who were not interviewed for the study. Two psychiatrists independently reviewed all available information including the DIGS, the FIGS, hospital records and the interviewer's notes. Best estimate lifetime psychiatric diagnosis according to the DSM-IV criteria were determined independently; if both psychiatrists disagreed about a diagnosis, a third one was sought and a consensus diagnosis was reached after discussion.

\subsection{2. $C P T$}

A CPT machine from Sunrise Systems, v. 2.20 (Pembroke, MA, USA), was used to assess sustained attention with a procedure detailed elsewhere (Chen et al., 1998a). Briefly, numbers from 0 to 9 were randomly presented for $50 \mathrm{~ms}$ each, at a rate of one per second for two sessions. During the first session (undegraded), subjects responded to the target stimulus (the number 9 preceded by the number 1) by pressing a button, whereas a pattern of snow was used to toggle background and foreground so that the image was visually distorted during the second session (25\% degraded). A total of 341 trials, $31(10 \%)$ of which were target stimuli, were presented over $5 \mathrm{~min}$ for each session. Both sessions include a working memory component and additional perceptual load is assessed in the degraded one (Nuechterlein et al., 1992).

The signal-detection indexes of performance on the test, sensitivity $\left(d^{\prime}\right)$ and response criterion $(\ln \beta)$, were derived from the hit rate and false-alarm rate (Nuechterlein, 1991; Swets, 1973). Sensitivity measures an individual's ability to discriminate target stimuli from nontarget stimuli, whereas response criterion assesses the amount of perceptual evidence an individual requires prior to making a decision to respond to a stimulus as a signal. Using the technique of Receiver Operating Characteristic analysis, the effect of decision criterion can be isolated from the sensitivity, and measures of $d^{\prime}$ and $\beta$ would be independent under the assumptions of normal and equal-variance distributions (Swets, 1973). Variables extraneous to the intended comparison of sensitivity, such as differences in motivation or in cooperativeness between the groups, would yield differences in $\ln \beta$ rather than in $d^{\prime}$.

A 1-week test-retest reliability study of the CPT versions used in this study was conducted in a separate sample of 10 schizophrenia inpatients, 10 nonpsychotic inpatients, and 10 normal age- and sex-matched control subjects (Chen et al., 1998a). The intraclass correlation coefficients of reliabilities (ICCRs) of CPT performance indices were reported for all participants together so the range of variation would be large enough to make a stable estimate. The ICCRs of $d^{\prime}$ were 0.83 (undegraded) and 0.82 (degraded), and those of $\ln \beta$ were 0.49 (undegraded) and 0.72 (degraded). A possible reason for a higher reliability of $\ln \beta$ for the degraded CPT than that for the undegraded one might be that the distraction caused by the degrading forced some participants to adopt a more stringent response criterion during the test. The CPT performance in a representative sample of 345 community subjects, whose sampling was described in detail previously (Chen et al., 1998a), was used as normative data for the standardization of subjects' CPT score in this study.

\subsection{Relatives: psychiatric status and exclusion criteria}

Among 199 first-degree relatives of the simplex and 143 of the multiplex families that completed at least one version of the CPT, 197 of the simplex and 123 of the 
multiplex were directly interviewed with the DIGS. No relatives in the simplex group had schizophrenia or schizophreniform disorder, while four of the multiplex group had schizophrenia and none had schizophreniform disorder. The two groups seemed to have comparable proportions of substance use disorders. In the simplex group, six (3.1\%) had alcohol abuse, three (1.5\%) alcohol dependence, one $(0.5 \%)$ drug abuse and one $(0.5 \%)$ drug dependence. The corresponding numbers in the multiplex group were $6(4.9 \%), 3$ $(2.4 \%), 0$ and $1(0.8 \%)$. Similar proportions of anxiety and mood disorders, except dysthymia, were also observed in the two groups. In the simplex group, 5 (2.5\%) had major depressive disorder, 14 (7.1\%) dysthymia, none bipolar disorder, obsessive-compulsive disorder or phobia, and $1(0.5 \%)$ panic disorder, while the corresponding numbers for the multiplex group were 3 (2.4\%), $2(1.6 \%), 0,1(0.8 \%), 0$ and 0 .

Any first-degree relatives with schizophrenia, schizophreniform disorder, alcohol/drug use disorders or mental retardation were excluded from the final analysis. Hence, 8 relatives for the simplex (7 with alcohol abuse or dependence, and 1 with alcohol and drug dependence) and 15 relatives for the multiplex families (4 with schizophrenia, 8 with alcohol abuse or dependence, 1 with alcohol and drug dependence, and 2 with mental retardation) were excluded from analysis. The final study sample included 107 nonpsychotic parents (from 74 families) and 84 nonpsychotic siblings (from 48 families) of schizophrenic probands from the simplex group (80 patients), and 72 nonpsychotic parents (from 54 families) and 56 nonpsychotic siblings (from 40 families) who were related to the multiplex group of schizophrenic probands (which included 166 co-affected patients from 99 families).

\subsection{Data analysis}

\subsubsection{Adjusted z score and perceptual load component for CPT performance}

The CPT scores were standardized with adjustments for sex, age and education as described previously (Chen et al., 1998b). The predictive score of a subject was calculated by using the regression coefficients obtained from the regression of the scores on the covariates among the 345 community subjects. The difference between the raw score and the predictive score was then standardized by the root mean error of the regression and was defined as the adjusted $z$ score of the subject. In addition, for three subjects younger than 16 years of age, the age-specific mean and standard deviation of a school sample (Lin et al., 1999) were used for standardization. Each subject was categorized on the basis of his or her adjusted $z$ scores of CPT $d^{\prime}$ against the cut-off points of $-1,-2$ and -2.5 .

In comparing the CPT performance of relatives from simplex families with that of relatives from multiplex families, the perceptual load component of the degraded CPT was assessed by regressing a relative's degraded score on his undegraded one. Then, the residual of the regression was treated as a proxy for the perceptual load processing. Because the perceptual load processing was found to be negatively correlated with age in the community sample $(r=-0.15, p=0.0046)$ and part of the current study ( $r=-0.22, p=0.0096$, for the siblings; $r=0.03, p=0.6968$, for the parents), an adjusted $z$ score for perceptual load processing was further calculated by including age in the regression of the degraded CPT $\mathrm{d}^{\prime}$ on the undegraded one.

\subsubsection{Statistical analysis}

Since some parents and siblings were from the same families, we adopted statistical models that allow for the adjustment for within-family correlation. For continuous response variables, linear mixed effect models with family as the random effect (Laird and Ware, 1982) was applied using the Proc MIXED procedure in the software package SAS version 8.2 for Windows (SAS Institute, Cary, NC, USA), whereas for categorical response variables, generalized estimating equations with family as the clustering variable (Zeger and Liang, 1986) were applied using the Proc GENMOD procedure. Effect sizes were calculated with the simplex group as the reference group to directly contrast the strength of evidence for genetic loading.

\section{Results}

The demographic features of the study subjects are shown in Table 1. The subjects from simplex and multiplex families, regardless of probands, parents or siblings, were comparable in terms of male-female ratio. Probands from simplex families were younger than those from multiplex families. In parallel, parents from simplex families were also younger than those from multiplex ones. Siblings from simplex families had fewer years of education than those from multiplex families.

Although all the first-degree relatives were invited to participate in the study, 1 out of $80(1 \%)$ simplex and 26 out of $99(26 \%)$ multiplex families failed to recruit any relatives for the assessment of the sustained attention. However, adjusted $z$ scores of CPT $d^{\prime}$ were not significantly different between the probands of the 
Table 1

Demographic characteristics of study subjects

\begin{tabular}{|c|c|c|c|c|c|c|c|}
\hline \multirow[t]{2}{*}{ Variable } & \multicolumn{2}{|l|}{ Probands } & \multicolumn{2}{|l|}{ Parents } & \multicolumn{2}{|l|}{ Siblings } & \multirow{2}{*}{$\begin{array}{l}\text { Community } \\
\text { sample } \\
(N=345)\end{array}$} \\
\hline & $\begin{array}{l}\text { Multiplex } \\
(N=166)\end{array}$ & $\begin{array}{l}\text { Simplex } \\
(N=80)\end{array}$ & $\begin{array}{l}\text { Multiplex } \\
(N=72)\end{array}$ & $\begin{array}{l}\text { Simplex } \\
(N=107)\end{array}$ & $\begin{array}{l}\text { Multiplex } \\
(N=56)\end{array}$ & $\begin{array}{l}\text { Simplex } \\
(N=84)\end{array}$ & \\
\hline Male, n (\%) & $100(60)$ & $43(54)$ & $29(40)$ & $41(38)$ & $28(50)$ & $40(48)$ & $165(48)$ \\
\hline $\begin{array}{l}\text { Age (years), } \\
\text { mean (S.D.) }\end{array}$ & $33.9(7.0)$ & $28.5^{\mathrm{a}}(7.3)$ & $59.6(7.9)$ & $56.2^{\mathrm{b}}(9.0)$ & $32.6(7.8)$ & $31.8(10.4)$ & $41.3(13.0)$ \\
\hline $\begin{array}{l}\text { Education (years) } \\
\text { mean (S.D.) }\end{array}$ & $11.8(2.9)$ & $11.4(3.0)$ & $7.1(4.6)$ & $7.6(4.8)$ & $13.3(2.2)$ & $12.3^{\mathrm{c}}(3.1)$ & $7.4(4.3)$ \\
\hline $\begin{array}{l}\text { Age at onset (years), } \\
\text { mean (S.D.) }\end{array}$ & $23.2(6.3)$ & $21.6(6.0)$ & & & & & \\
\hline
\end{tabular}

${ }^{\mathrm{a}} p<0.001(t=-5.61, d f=244)$ for the difference between simplex and multiplex groups.

b $p<0.05(t=-2.59, d f=177)$ for the difference between simplex and multiplex groups.

c $p<0.05(t=-2.21, d f=138)$ for the difference between simplex and multiplex groups.

multiplex families with relatives' participation and those without $(p=0.19$ for the undegraded and 0.70 for the degraded CPT). Among the participating families, the mean number of siblings per family differed signifi- cantly between the multiplex 4.6 (S.D. 1.9) and simplex 3.6 (S.D. 1.6) groups $(t=3.79, d f=178, p=0.0002)$.

The performance scores on the CPT by first-degree relatives are listed in Table 2. No significant differences

Table 2

The scores of the Continuous Performance Test (CPT) in nonpsychotic first-degree relatives of schizophrenic probands and community sample

\begin{tabular}{|c|c|c|c|c|c|c|c|c|c|}
\hline \multirow[t]{3}{*}{ CPT score } & \multicolumn{2}{|l|}{ Parents } & \multirow{3}{*}{$\begin{array}{l}\text { Effect } \\
\text { size }\end{array}$} & \multirow{3}{*}{$\begin{array}{l}\text { Group } \\
\text { comparison }^{\mathrm{a}} \\
p\end{array}$} & \multicolumn{2}{|l|}{ Siblings } & \multirow{3}{*}{$\begin{array}{l}\text { Effect } \\
\text { size }\end{array}$} & \multirow{3}{*}{$\begin{array}{l}\text { Group } \\
\text { comparison }^{\mathrm{a}} \\
p\end{array}$} & \multirow{3}{*}{$\begin{array}{l}\text { Community } \\
\text { sample } \\
(n=345) \\
\text { Mean (S.D.) }\end{array}$} \\
\hline & \multirow{2}{*}{$\begin{array}{l}\begin{array}{l}\text { Multiplex } \\
\left(n=72^{\mathrm{b}}\right)\end{array} \\
\text { Mean (S.D.) }\end{array}$} & \multirow{2}{*}{$\begin{array}{l}\text { Simplex } \\
\left(n=107^{\mathrm{b}}\right) \\
\text { Mean (S.D.) }\end{array}$} & & & \multirow{2}{*}{$\begin{array}{l}\begin{array}{l}\text { Multiplex } \\
(n=56)\end{array} \\
\text { Mean (S.D.) }\end{array}$} & \multirow{2}{*}{$\begin{array}{l}\text { Simplex } \\
\left(n=84^{\mathrm{b}}\right)\end{array}$} & & & \\
\hline & & & & & & & & & \\
\hline \multicolumn{10}{|l|}{ Raw score } \\
\hline $\begin{array}{l}\operatorname{Ln} \beta \text { on the } \\
\text { undegraded test }\end{array}$ & $1.7^{\mathrm{c}}(1.6)$ & $2.0(1.5)$ & -0.2 & 0.29 & $2.5(0.9)$ & $2.2(1.0)$ & 0.3 & 0.48 & $2.2(0.9)$ \\
\hline $\begin{array}{c}\operatorname{Ln} \beta \text { on the } 25 \% \\
\text { degraded test }\end{array}$ & $1.4^{\mathrm{c}}(1.5)$ & $1.4^{\mathrm{c}}(1.8)$ & 0 & 0.99 & $1.8^{\mathrm{d}}(1.2)$ & $2.1(1.3)$ & -0.2 & 0.05 & $2.2(0.9)$ \\
\hline $\begin{array}{l}d^{\prime} \text { on the undegraded } \\
\text { test }\end{array}$ & $3.0^{\mathrm{c}}(1.5)$ & $3.2^{\mathrm{c}}(1.5)$ & -0.1 & 0.43 & $3.6^{\mathrm{c}}(1.2)$ & $3.7(1.2)$ & -0.1 & 0.20 & $4.0(0.8)$ \\
\hline $\begin{array}{l}d^{\prime} \text { on the } 25 \% \\
\text { degraded test }\end{array}$ & $2.1^{\mathrm{c}}(1.5)$ & $2.2^{\mathrm{c}}(1.7)$ & -0.1 & 0.66 & $2.5^{\mathrm{c}}(1.5)$ & $3.1(1.5)$ & -0.4 & 0.01 & $3.3(1.0)$ \\
\hline $\begin{array}{l}\text { Perceptual load } \\
\text { processing }\end{array}$ & $0.0(1.1)$ & $0.0(1.1)$ & 0 & 0.77 & $-0.3(1.0)$ & $0.2(0.9)$ & -0.6 & $<0.01$ & $0.0(0.8)$ \\
\hline \multicolumn{10}{|l|}{ Adjusted z score } \\
\hline $\begin{array}{l}\operatorname{Ln} \beta \text { on the } \\
\quad \text { undegraded test }^{\mathrm{e}}\end{array}$ & $-0.8^{\mathrm{c}}(1.9)$ & $-0.4^{\mathrm{c}}(1.8)$ & -0.2 & 0.24 & $0.5^{\mathrm{d}}(1.1)$ & $0.2(1.2)$ & 0.3 & 0.51 & $0(1.0)$ \\
\hline $\begin{array}{l}\operatorname{Ln} \beta \text { on the } 25 \% \\
\text { degraded test }^{\mathrm{e}}\end{array}$ & $-0.9^{\mathrm{c}}(1.7)$ & $-0.9^{\mathrm{c}}(1.9)$ & 0 & 1.00 & $-0.5^{\mathrm{d}}(1.3)$ & $-0.0(1.5)$ & -0.3 & 0.05 & $0(1.0)$ \\
\hline $\begin{array}{l}d^{\prime} \text { on the undegraded } \\
\text { test }^{\mathrm{e}}\end{array}$ & $-0.9^{\mathrm{c}}(2.0)$ & $-0.8^{\mathrm{c}}(2.0)$ & -0.1 & 0.60 & $-1.2^{\mathrm{c}}(1.6)$ & $-0.9^{c}(1.6)$ & -0.2 & 0.14 & $0(1.0)$ \\
\hline $\begin{array}{l}d^{\prime} \text { on the } 25 \% \\
\text { degraded test }^{\mathrm{e}}\end{array}$ & $-0.9^{\mathrm{c}}(1.6)$ & $-0.9^{\mathrm{c}}$ & 0 & 0.87 & $-1.5^{\mathrm{c}}(1.6)$ & $-0.7^{\mathrm{c}}(1.6)$ & -0.5 & $<0.01$ & $0(1.0)$ \\
\hline $\begin{array}{l}\text { Perceptual load } \\
\text { processing }\end{array}$ & $0.3(1.4)$ & $0.2(1.3)$ & 0.1 & 0.65 & $-0.5^{\mathrm{c}}(1.2)$ & $0.1(1.1)$ & -0.5 & $<0.01$ & $0(1.0)$ \\
\hline
\end{tabular}

\footnotetext{
${ }^{a}$ Linear mixed effect model with family as the random effect to control for intrafamilial correlation.

${ }^{\mathrm{b}}$ Four multiplex parents, five simplex parents and two simplex siblings missing for the degraded test

${ }^{c} p<0.01$ vs. community sample, using linear mixed effect model with family as the random effect.

d $p<0.05$ vs. community sample, using linear mixed effect model with family as the random effect.

e Adjusted for sex, age and education level.

${ }^{\mathrm{f}}$ Adjusted for age.
} 
were observed between parents from simplex families and those from multiplex families in both the undegraded and degraded CPT $\ln \beta$ and $d^{\prime}$, and hence in perceptual load processing. In contrast, siblings from multiplex families had a lower mean $\ln \beta$ and $d^{\prime}$, both raw and adjusted $z$ scores, than their counterparts from simplex families on the degraded CPT. When the effect of perceptual load was separated from the working memory-related CPT performance, siblings from multiplex families had lower proficiency in processing the perceptual load as compared with siblings from simplex families. Similar results were found on effect sizes, with the greatest differences between the two groups being the degraded $\mathrm{d}^{\prime}$ and perceptual load for the siblings.

When compared with the community sample, the parents and siblings of both multiplex and simplex families showed lower mean CPT $d^{\prime}$ after adjusting for sex, age, and education level (Table 2). However, perceptual load processing did not discriminate the relatives of schizophrenic patients from the community sample. Parents in both groups of schizophrenia also showed lower $\ln \beta$ than the community sample, whereas $\ln \beta$ was equivalent between the siblings in the simplex group and the community sample. Additionally, siblings from multiplex families even showed a higher mean $\ln \beta$ than the community sample.

Similarly, using a categorical index to indicate impairment in sustained attention, the differences in the proportion of subjects with CPT deficit between simplex and multiplex families were only significant for the siblings on the degraded version (Table 3). For the three arbitrarily chosen cut-off points in the adjusted $z$ score of degraded CPT $d^{\prime}$, the contrast between the two groups of the siblings was significant for the cut-off points of -1 or -2 , but only borderline for that of -2.5 . Nevertheless, the risk ratio of the multiplex group to the simplex one at the cut-off point of -2.5 (1.89) was similar to that for the cut-off point of $-2(1.95)$ or -1 (1.56).

\section{Discussion}

To our knowledge, this is the first study comparing sustained attention in nonpsychotic relatives of schizophrenic patients with different familial loading for schizophrenia. There have been two major criticisms of grouping patients according to family history in schizophrenia research (Goldin et al., 1987). First, the family history negative families might still carry the genetic liability. If the number of relatives is small or information is not available on some relatives, the type of family history could be misclassified. Second, the question of whether a relative is affected depends on the disorders incorporated in the definition of schizophrenia and whether these are genetically linked to schizophrenia, and hence is open to debate. Therefore, Lewis et al. (1987) suggested using large sample sizes and taking a complete family history when using the familialsporadic approach.

With the above criticisms in mind, we adopted a grouping strategy with a sample size larger than previous studies (Lencer et al., 2000, 2003; Ross et al., 1998) and stringent definitions of familiality and sporadicity. In addition, other methodological concerns, including use of personal interviews for the diagnosis of relatives and controlling for confounding factors (Roy and Crowe, 1994), were also addressed in this study. However, we still failed to see a difference in sustained

Table 3

Number and proportion of relatives whose adjusted $z$ score of $d^{\prime a}$ on the Continuous Performance Test (CPT) was below a threshold

\begin{tabular}{|c|c|c|c|c|c|c|c|c|}
\hline \multirow{3}{*}{$\begin{array}{l}\text { Cut-off } \\
\text { in } \\
\text { adjusted } \\
z \text { score }^{\text {a }}\end{array}$} & \multicolumn{2}{|l|}{ Parents } & \multirow{2}{*}{\multicolumn{2}{|c|}{$\begin{array}{l}\text { Group } \\
\text { comparison }^{\mathrm{b}}\end{array}$}} & \multicolumn{2}{|l|}{ Siblings } & \multicolumn{2}{|c|}{ Group comparison ${ }^{\mathrm{b}}$} \\
\hline & \multirow{2}{*}{$\frac{\text { Multiplex }\left(n=72^{\mathrm{c}}\right)}{N(\%)}$} & \multirow{2}{*}{$\frac{\text { Simplex }\left(n=107^{\mathrm{c}}\right)}{N(\%)}$} & & & \multirow{2}{*}{$\frac{\text { Multiplex }(n=56)}{N(\%)}$} & \multirow{2}{*}{$\frac{\text { Simplex }\left(n=84^{\mathrm{c}}\right)}{N(\%)}$} & & \\
\hline & & & $\chi^{2}(d f=1)$ & $p$ & & & $\chi^{2}(d f=1)$ & $p$ \\
\hline \multicolumn{9}{|c|}{ Undegraded version } \\
\hline$<-1$ & $28(38.9)$ & 37 (34.6) & 0.23 & 0.63 & $24(42.9)$ & $27(32.1)$ & 2.33 & 0.13 \\
\hline$<-2$ & $15(20.8)$ & $21(19.6)$ & 0.09 & 0.77 & $15(26.8)$ & $22(26.2)$ & 0.13 & 0.72 \\
\hline$<-2.5$ & $12(16.7)$ & $16(15.0)$ & 0.09 & 0.76 & $10(17.9)$ & $15(17.9)$ & 0.19 & 0.66 \\
\hline \multicolumn{9}{|c|}{ Degraded version } \\
\hline$<-1$ & $28(41.2)$ & $45(44.1)$ & 0.15 & 0.70 & $34(60.7)$ & $32(39.0)$ & 6.76 & $<0.01$ \\
\hline$<-2$ & $17(25.0)$ & $21(20.6)$ & 0.30 & 0.58 & $20(35.7)$ & $15(18.3)$ & 5.29 & 0.02 \\
\hline$<-2.5$ & $12(17.7)$ & 19 (18.6) & 0.08 & 0.78 & $13(23.2)$ & $10(12.2)$ & 3.14 & $0.08^{\mathrm{d}}$ \\
\hline
\end{tabular}

a Adjusted for sex, age, and education level.

b Score statistics for type 3 GEE analysis, correlation structure: exchangeable.

c Four multiplex parents, five simplex parents, and two simplex siblings missing for the degraded test.

${ }^{\mathrm{d}} Z=1.95, p=0.05$, analysis of GEE parameter estimates, empirical standard error estimates. 
attention between the parents from simplex and multiplex families, and even the siblings of the two groups differed only on the degraded CPT. Several reasons might account for this. First, according to quantitative genetic theory (Falconer and Mackay, 1996), siblings share one fourth non-additive genetic variance, which is not shared by a parent-offspring pair. If there is a non-additive genetic component of schizophrenia susceptibility and sustained attention is indeed part of it, we would see a sharper contrast between simplex and multiplex siblings than between simplex and multiplex parents. Second, since siblings share more in both pre- and post-natal environment than parent-offspring do, our results might also suggest the contribution of "environmental loading" to sustained attention. Third, the siblings of schizophrenic patients tend to have higher risk for schizophrenia, hence for carrying the genetic susceptibility, than the parents because of the reduced fertility effect of schizophrenia (Kendler and MacLean, 1989). Fourth, the tasks performed on the CPT might involve different cognitive components, to which genes might contribute differentially. In this study, the degraded version of the CPT appears to be a more sensitive indicator of genetic susceptibility to schizophrenia than the undegraded one. Another interesting issue was related to the fact that multiplex was defined as having a sibling with schizophrenia in the current study and the familiality effect was observed in siblings only. Whether the familiality effect could be found in parents if multiplex had been defined as having a parent with schizophrenia deserves further investigation. By examining the role of ascertainment bias on the familiality effect of sustained attention in schizophrenia families, the impact of sibling- or parentspecific genetic or environmental factors might possibly be clarified.

The worse performance of siblings from multiplex families on the degraded CPT was accompanied by a significant decrease in $\ln \beta$. However, $\ln \beta$ did not appear to be consistently associated with $d^{\prime}$ in this study. Siblings from simplex families were comparable to the community sample in $\ln \beta$ but showed lower $d^{\prime}$ on both undegraded and degraded CPT after adjusting for sex, age and educational level. Therefore, the dissociation between $d^{\prime}$ and $\ln \beta$ was consistent with the expectation of signal detection theory. The effect of motivation should at best partially explain the worse performance on the CPT.

Among various versions of the CPT, the " $1-9$ " task used in this study includes a working memory component in both the undegraded and the degraded versions and additional perceptual load in the degraded one (Nuechterlein et al., 1992). Our results are consistent with previous family studies in the sense that, among a battery of cognitive tests, only the one involving visual working memory was shown to be worse in families with two or more schizophrenic patients than in families with sporadic cases (Faraone et al., 2000; Tuulio-Henriksson et al., 2003). However, working memory measured by different tasks may have more than one dimension (Pukrop et al., 2003). Thus, the issue of whether working memory with and without perceptual load is a unitary construct is worth examining.

In previous studies using more difficult CPT versions, the performance deficits in schizophrenic patients were more significant (Seidman et al., 1998) and not amenable to short-term treatment with neuroleptics (Epstein et al., 1996; Liu et al., 2000). Moreover, after the effect of demographic features was adjusted for, the degraded version of the CPT led to a higher recurrence risk ratio in nonpsychotic relatives of schizophrenic patients than the undegraded one (Chen et al., 2004). In line with these findings, this study provides further evidence that the degraded CPT is more sensitive than the undegraded one in distinguishing the nonpsychotic relatives of the simplex families from those of the multiplex ones.

One unique feature of this study is that the residual of the regression of the degraded score on the undegraded one was used to differentiate the effect of perceptual load from that of working memory load on the task of the degraded CPT. Our results indicate that siblings from multiplex families indeed were less proficient than their counterparts from simplex families in processing the perceptual load. Although the working memory component of the CPT, as indicated by the undegraded CPT score, can differentiate schizophrenic patients from normal controls (Chen et al., 1998b), this finding suggests that it is the add-on perceptual load in the degraded CPT that accounts for the better discrimination and for the higher genetic susceptibility in the nonpsychotic siblings of multiplex families.

Unlike the finding on the recurrence risk ratio of the CPT deficits (Chen et al., 2004), increased stringency in the cut-off point in the degraded CPT did not lead to a better discrimination between siblings from simplex and multiplex families. One possible reason for this is that the optimal threshold for the difference in CPT deficit between the first-degree relatives of schizophrenic patients and normal controls might differ from that for the difference in CPT deficit between siblings from multiplex and simplex families. 
Some limitations of this study should be kept in mind in interpreting our results. First, the relatively low participation rate of the relatives in the multiplex families might bias the mean CPT performance in this group of relatives. Although there were no significant differences in the CPT performance between the probands of the families with and without relatives' participation, we could not rule out the possibility that the relatives of the multiplex families without any relative's participation turned out to have more severe attention deficit. If so, the CPT performance in the relatives of the multiplex families would be biased toward better performance and hence not be different from that in the relatives of the simplex families. Second, because we interviewed directly first-degree relatives only, the schizophrenic probands of the simplex families in this study might have a seconddegree relative with schizophrenia. However, the impact of this misclassification would be relatively minor since morbidity risks of schizophrenia among uncles/aunts and nieces/nephews were only $2 \%$ and $4 \%$ (Tsuang, 2000), respectively. Third, with a smaller family size and a mean age of 32 years for the siblings of the simplex families, some families might be misclassified owing to censoring bias. Nevertheless, all of the above limitations would diminish rather than increase the simplex-multiplex distinction.

In summary, this study indicates that the simplexmultiplex distinction in sustained attention is useful for the siblings but not for the parents of schizophrenic patients. Siblings from multiplex families exhibited worse performance on the degraded CPT and less proficiency in processing the perceptual load than did their counterparts from simplex families. These findings further support the claim that impairment in sustained attention along with perceptual load processing is a sensitive endophenotypic marker for schizophrenia and might be useful in future genetic dissection.

\section{Acknowledgments}

This study was supported by grants from the National Health Research Institutes, Taiwan (DOH 88-HR-825, NHRI-GT-EX89P825P，NHRI-EX90-8825PP，NHRIEX91-9113PP, NHRI-EX92-9113PP) and National Science Council, Taiwan (NSC83-0412-B-002-310, NSC84-2331-B-002-187). The authors thank Drs. Ching-Jui Chang, Hung-Jung Chang, Joseph J. Cheng, Shi-Chin Guo, Hai Ho, Hsien-Yuan Lane, Shi-Kwang Lin, Wen-Chen Ou Yang, Fu-Chuan Wei and Tien-Wei Yang for their help in the recruitment and evaluation of schizophrenic patients.

\section{References}

Almasy, L., Blangero, J., 2001. Endophenotypes as quantitative risk factors for psychiatric disease: rationale and study design. American Journal of Medical Genetics 105, 42-44.

Braff, D.L., 1993. Information processing and attention dysfunctions of schizophrenia. Schizophrenia Bulletin 19, 233-259.

Chang, C.-J., Chen, W.J., Liu, S.-K., Cheng, J.J., Ou Yang, W.-C., Chang, H.-J., Lane, H.-Y., Lin, S.-K., Yang, T.-W., Hwu, H.-G., 2002. Morbidity risk of psychiatric disorders among the first degree relatives of schizophrenic patients in Taiwan. Schizophrenia Bulletin 28, 379-392.

Chen, W.J., Faraone, S.V., 2000. Sustained attention deficits as markers of genetic susceptibility to schizophrenia. American Journal of Medical Genetics (Seminars in Medical Genetics) 97, 52-57.

Chen, W.J., Hsiao, C.K., Hsiao, L.-L., Hwu, H.-G., 1998a. Performance of the Continuous Performance Test among community samples. Schizophrenia Bulletin 24, 163-174.

Chen, W.J., Liu, S.K., Chang, C.-J., Lien, Y.-J., Chang, Y.-H., Hwu, H.-G., 1998b. Sustained attention deficit and schizotypal personality features in nonpsychotic relatives of schizophrenic patients. American Journal of Psychiatry 155, 1214-1220.

Chen, W.J., Chang, C.-H., Liu, S.K., Hwang, T.J., Hwu, H.-G., Collaborators from the Multidimensional Psychopathology Group Research Project, 2004. Sustained attention deficits in nonpsychotic relatives of schizophrenic patients: a recurrence risk ratio analysis. Biological Psychiatry 55, 995-1000.

Cornblatt, B.A., Keilp, J.G., 1994. Impaired attention, genetics, and the pathophysiology of schizophrenia. Schizophrenia Bulletin 20, 31-46.

DeLisi, L.E., Mesen, A., Rodriguez, C., Bertheau, A., LaPrade, B., Llach, M., Riondet, S., Razi, K., 2001. Clinical characteristics of schizophrenia in multiply affected Spanish origin families from Costa Rica. Psychiatric Genetics 11, 145-152.

Epstein, J.I., Keefe, R.S.E., Roitman, S.L., Harvey, P.D., Mohs, R.C., 1996. Impact of neuroleptic medications on continuous performance test measures in schizophrenia. Biological Psychiatry 39, 902-905.

Falconer, D.S., Mackay, T.F.C., 1996. Introduction to Quantitative Genetics, 4th edn. Longman, Harlow.

Faraone, S.V., Seidman, L.J., Kremen, W.S., Toomey, R., Pepple, J.R., Tsuang, M.T., 2000. Neuropsychologic functioning among the nonpsychotic relatives of schizophrenic patients: the effect of genetic loading. Biological Psychiatry 48, 120-126.

Farmer, A., McGuffin, P., Gottesman, I.I., 1990. Problems and pitfalls of the family history positive and negative dichotomy: response to Dalen. Schizophrenia Bulletin 16, 367-370.

Goldin, L.R., DeLisi, L.E., Gershon, E.S., 1987. Unravelling the relationship between genetic and environmental risk factors in psychiatric disorders. British Journal of Psychiatry 151, 302-305.

Gottesman, I.I., Gould, T.D., 2003. The endophenotype concept in psychiatry: etymology and strategic intentions. American Journal of Psychiatry 160, 636-645.

Kendler, K.S., 1987. The impact of diagnostic misclassification on the pattern of familial aggregation and coaggregation of psychiatric illness. Journal of Psychiatric Research 21, 55-91.

Kendler, K.S., 1988. The sporadic v. familial classification given aetiological heterogeneity: II. Power analyses. Psychological Medicine 18, 991-999.

Kendler, K.S., MacLean, C.J., 1989. The impact of altered fitness on the risk of illness in relatives. Genetic Epidemiology 6, 481-491. 
Laird, N.M., Ware, J.H., 1982. Random-effects models for longitudinal data. Biometrics 38, 963-974.

Lencer, R., Malchow, C., Trillenberg-Krecker, K., Schwinger, E., Arolt, V., 2000. Eye-tracking dysfunction (ETD) in families with sporadic and familial schizophrenia. Biological Psychiatry 47, 391-401.

Lencer, R., Trillenberg-Krecker, K., Schwinger, E., Arolt, V., 2003. Schizophrenia spectrum disorders and eye tracking dysfunction in singleton and multiplex schizophrenia families. Schizophrenia Research 60, 33-45.

Lewis, S.W., Reveley, A.M., Reveley, M.A., Chitkara, B., Murray, R. M., 1987. The familial/sporadic distinction as a strategy in schizophrenia research. British Journal of Psychiatry 151, 306-313

Lin, C.C.H., Hsiao, C.K., Chen, W.J., 1999. Development of sustained attention assessed using the Continuous Performance Test among children 6-15 years of age. Journal of Abnormal Child Psychology 27, 403-412.

Liu, S.K., Chen, W.J., Chang, C.-J., Lin, H.-N., 2000. Effects of atypical neuroleptics on sustained attention deficits in schizophrenia: a trial of risperidone versus haloperidol. Neuropsychopharmacology 22, 311-319.

NIMH Genetics Initiative, 1992a. Diagnostic Interview for Genetic Studies. National Institute of Mental Health, Rockville, MD.

NIMH Genetics Initiative, 1992b. Family Interview for Genetic Studies. National Institute of Mental Health, Rockville, MD.

Nuechterlein, K.H., 1991. Vigilance in schizophrenia and related disorders. In: Steinhauer, S.R., Gruzelier, J.H., Zubin, J. (Eds.), Neuropsychology, Psychophysiology and Information Processing. Handbook of Schizophrenia, vol. 5. Elsevier, Amsterdam, pp. 397-433.

Nuechterlein, K.H., Dawson, M.E., 1984. Information processing and attentional functioning in the developmental course of schizophrenic disorders. Schizophrenia Bulletin 10, 160-203.

Nuechterlein, K.H., Dawson, M.E., Gitlin, M., Ventura, J., Goldstein, M.J., Snyder, K.S., Yee, C.M., Mintz, J., 1992. Developmental processes in schizophrenic disorders: longitudinal studies of vulnerability and stress. Schizophrenia Bulletin 18, $387-425$

Nurnberger Jr., J.I., Blehar, M.C., Kaufmann, C.A., York-Cooler, C., Simpson, S.G., Harkavy-Friedman, J., Severe, J.B., Malaspina, D., Reich, T., 1994. Diagnostic interview for genetic studies.
Rationale, unique features, and training. NIMH Genetics Initiative. Archives of General Psychiatry 51, 849-859.

Pukrop, R., Matuschek, E., Ruhrmann, S., Brockhaus-Dumke, A., Tendolkar, I., Bertsch, A., Klosterkotter, J., 2003. Dimensions of working memory dysfunction in schizophrenia. Schizophrenia Research 62, 259-268.

Ross, R.G., Olincy, A., Harris, J.G., Radant, A., Adler, L.E., Freedman, R., 1998. Anticipatory saccades during smooth pursuit eye movement and familial transmission of schizophrenia. Biological Psychiatry 44, 690-697.

Rosvold, H.E., Mirsky, A.F., Sarason, I., Bransome Jr., E.D., Beck, L. H., 1956. A Continuous Performance Test of brain damage. Journal of Consulting Psychology 20, 343-350.

Roy, M.A., Crowe, R.R., 1994. Validity of the familial and sporadic subtypes of schizophrenia. American Journal of Psychiatry 151, 805-814.

Roy, M.A., Flaum, M.A., Gupta, S., Jaramillo, L., Andreasen, N.C., 1994. Epidemiological and clinical correlates of familial and sporadic schizophrenia. Acta Psychiatrica Scandinavica 89, 324-328.

Seidman, L.J., Van Manen, K.-J., Turner, W.M., Gamser, D.M., Faraone, S.V., Goldstein, J.M., Tsuang, M.T., 1998. The effects of increasing resource demand on vigilance performance in adults with schizophrenia or developmental attentional/learning disorders: a preliminary study. Schizophrenia Research 34, 101-112.

Swets, J., 1973. The relative operating characteristic in psychology: a technique for isolating effects of response bias finds wide use in the study of perception and cognition. Science 182, 990-1001.

Tsuang, M., 2000. Schizophrenia: genes and environment. Biological Psychiatry 47, 210-220.

Tsuang, M.T., Lyons, M.J., Faraone, S.V., 1990. Heterogeneity of schizophrenia: conceptual models and analytic strategies. British Journal of Psychiatry 156, 17-26.

Tuulio-Henriksson, A., Arajarvi, R., Partonen, T., Haukka, J., Varilo, T., Schreck, M., Cannon, T., Lonnqvist, J., 2003. Familial loading associates with impairment in visual span among healthy siblings of schizophrenia patients. Biological Psychiatry 54, 623-628.

Walker, E., Shaye, J., 1982. Familial schizophrenia. A predictor of neuromotor and attentional abnormalities in schizophrenia Archives of General Psychiatry 39, 1153-1156.

Zeger, S.L., Liang, K.Y., 1986. Longitudinal data analysis for discrete and continuous outcomes. Biometrics 42, 121-130. 